\title{
$\mathrm{CiSj}$
}

\section{SMART LICENSE PLATE RECOGNITION SYSTEM BASED ON IMAGE PROCESSING USING NEURAL NETWORK}

\author{
V. Koval ${ }^{1)}$, V. Turchenko ${ }^{1)}$, V. Kochan ${ }^{1)}$, A. Sachenko ${ }^{1)}$, G. Markowsky ${ }^{2)}$ \\ 1) Ternopil Academy of National Economy, Institute of Computer Information Technologies, \\ 3 Peremoga Square, 46004, Ternopil, Ukraine, e-mail: vko@tanet.edu.te.ua \\ 2) Department of Computer Science, 5752 Neville Hall, University of Maine, Orono, \\ ME 04469-5752, e-mail: markov@cs.umaine.edu
}

\begin{abstract}
This paper describes the Smart Vehicle Screening System, which can be installed into a tollbooth for automated recognition of vehicle license plate information using a photograph of a vehicle. An automated system could then be implemented to control the payment of fees, parking areas, highways, bridges or tunnels, etc. There are considered an approach to identify vehicle through recognizing of it license plate using image fusion, neural networks and threshold techniques as well as some experimental results to recognize the license plate successfully.
\end{abstract}

Keywords: - Homeland security, License plate recognition, Artificial intelligence

\section{INTRODUCTION}

The task of vehicle identification can be solved by vehicle license plate recognition. It can be used in many applications such as entrance admission, security, parking control, airport or harbor cargo control, road traffic control, speed control and so on. A number of commercial software is developed in this area. However, they cannot be readily used when vehicle image is provided in different styles and formats [1-3]. Proposed approach allows removing this drawback by ensemble of two methods: (i) detection and extraction of image region included license plate from source images flow and (ii) recognition of character presented on the license plate.

Image processing techniques such as edge detection, thresholding and resampling have been used to locate and isolate the license plate and the characters. The neural network was used for successful recognition the license plate number. Once a license plate has been accurately identified, information about the vehicle can be obtained from various databases. Should the information suggest that there is anything suspicious about the vehicle, appropriate actions can be taken.

The algorithm of license plate recognition (LPR) consists of the following steps: (i) to capture the car's images, (ii) to deblur of image frames, (iii) to extract image of license plate, (iv) to extract characters from license plate image, (v) to recognize license plate characters and identify the vehicle.

\section{DEBLURRING TECHNOLOGY AND IMAGE FUSION}

Capture of the images.

We investigated 8-bit grayscale images captured with a digital camera and transferred to computer. Our images consist of rectangular array 480x640 pixels. In order to receive the better image for further processing it is supposed to receive video flow included several grabbed frames in the same time with different focus.

Deblurring of image frames.

We consider situations where two or more objects in the scene are at different distances from the camera. It is difficult to receive fully focused image using inexpensive cameras. Obtained images are not in focus everywhere, i.e., if one object is in focus, another one will be out of focus. Despite this it is possible to enhance image quality which objects in focus everywhere using images fusion techniques with different focus points. Image fusion refers to image processing techniques to produce a new enhanced image by combination images from two or more cameras [4]. The fused image is then more suitable for further image-processing tasks such as segmentation, feature extraction and object recognition.

The simplest image fusion method just takes the pixel-by-pixel gray level average of source images. However, this often leads to undesirable side effects such as reduced contrast. The various methods based on the multiscale transforms have been proposed to address this issue. The basic idea is to perform a 
multiresolution decomposition on each source image, then integrate all these decompositions to form a composite representation, and finally reconstruct the fused image by performing an inverse multiresolution transform. Examples of this approach include the Laplacian pyramid [5], the gradient pyramid [6], the ratio-of-low-pass pyramid [7], the morphological pyramid [8], and the wavelet transform $[4,9,10]$. We propose the image fusion method based on mean contrast frequency. This method is computationally simple and can be used in real-time applications.

\section{Image fusion.}

The main purpose on this step is to receive as more deblurred image as possible, taking into account different weather conditions, possible movement of the vehicle and noises influences on the image capture procedure. The image fusion algorithm includes the following steps:

1. to create the MxN image template from source image with grayscale value $\operatorname{PICT}(m, n)$. Such template is shifted by the horizontal and vertical directions of both images.

2. to calculate the mean contrast frequency value for each position of template:

$$
\begin{gathered}
T F=|R F+C F| ; \\
R F=\frac{\sum_{m=1}^{M} \sum_{n=1}^{N}|\operatorname{PICT}(m, n)-\operatorname{PICT}(m+1, n)|}{(M-1) *(N-1)} ; \\
C F=\frac{\sum_{m=1}^{M} \sum_{n=1}^{N}|\operatorname{PICT}(m, n)-\operatorname{PICT}(m, n+1)|}{(M-1) * N(-1)},
\end{gathered}
$$

where $\operatorname{PICT}(m, n)$ is color value of template's pixel located at $\mathrm{m}$ - row and $\mathrm{n}$ - column; $\mathrm{M}, \mathrm{N}$ are size of template. The template frequencies are calculated for each of both blurred images.

3. to create each pixel of deblurred image by thresholding procedure:

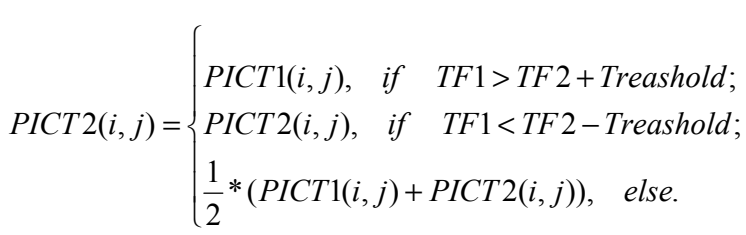

where PICT1(i,j) and PICT2(i,j) are pixels located at the center of templates received from fused source images.

In order to research the proposed approach, the different parts of image were artificially blurred using the linear motion of an image by 8 pixels, with an angle of 75 and 90 degrees respectively in a counter-clockwise direction (Fig. 1a and Fig. 1b). Also the image template with $5 \times 5$ pixels was used for image fusing process.
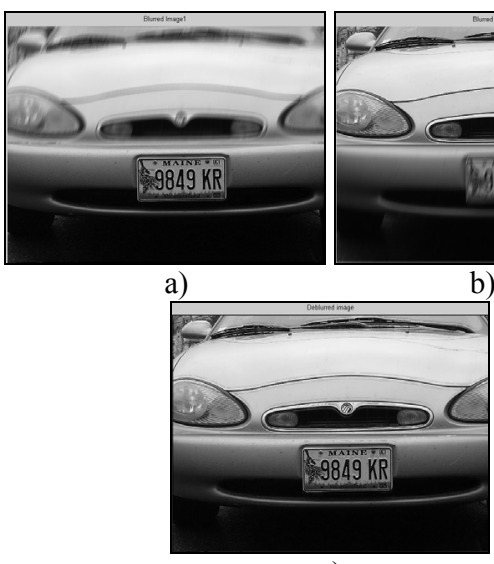

c)

Fig. 1 - Blurred images (a and b), and result of image fusion techniques (c).

Thresholding.

For determination the place that contains the license plate, the different objects presented in the image are founded and labeled. Those that have geometrical characteristics similar to those of license plate characters are selected. It is assumed that the plate has been found if the relative position of the selected objects is consistent with license plate. In a grayscale image the thresholding transformation sets each gray level that is less than or equal to a chosen threshold value $\mathrm{T}$ to 0 and each gray level greater than $\mathrm{T}$ to 1 (5). The result is $\mathrm{B} / \mathrm{W}$ image with 0 representing black and 1 representing white (Fig. 2). Received from the digital camera images are transformed into black and white binary images, where any color below the threshold value is set to black, while any color above the threshold value is set to white. The threshold level $\mathrm{T}$ depends highly on the lighting conditions and brightness of the area of source image. The chosen value was selected experimentally taking into account artificially generated lights of the possible checkpoint of the tollbooth.

$$
\operatorname{PICT3}(i, j)= \begin{cases}1, & \text { if } \operatorname{PICT2}(i, j)>\text { Treashold } \\ 0, & \text { else }\end{cases}
$$

\section{LICENSE PLATE IDENTIFICATION}

The most difficult task is to locate the license plate, which could be at anywhere in the image. This task becomes more challenging if the illumination of the

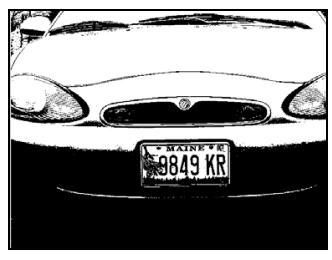

Fig. 2. - Thresholded binary image. 
image varies from one plate to another. There have been a number of studies in LPR $[1,2,3]$. One of them uses Hough Transform (HT) analysis of the image. Hough transformation is a method for detecting lines in binary images. It is possible to form the list of lines in the form of accumulator cells using such technique. These cells are then analyzed and list of horizontal and vertical line segments are combined for rectangular region selections. The candidate regions of license plate are received analyzing the length of previously received lines [1]. However, boundary line detection is not suitable completely in the case of not horizontally locating of the license plates on the image, corrupting or absentness of the boundary line in the license plates due to noise and uneven brightness. Furthermore, HT is inherently a time-consuming process [1].

Our LPR system uses an iterative thresholding operation to identify license plate of vehicle. To determine the regions of the image that contains the license plate, the different objects presented in the image are found and labeled using the connectivity reason of each pixel in matrix. The labeled objects are then analyzed and those that possess geometrical characteristics similar to license plate characters are selected. The characteristics we have used so far are height and width, but others, such as aspect ratio, are also possible. The license plate can be identifying using the following criteria: (i) Total pixels in a object, (ii) Height of the blobs, (iii) Width of the blobs, (iv) Presence license characters in the region.

The proportion of maximum width and high is calculated for each object. Then object is considered as a region, which can include license plate if boundary of proportion is from 0.1 to 0.25 and if total number of pixels in the object is more than 2000 and less than 8000 .

On the next step selected regions are inverted and renumbered (relabeled) due to the connectivity reason. Then such region objects are selected that include more than threshold number of white pixels (equal 1). The threshold shows minimum number of white pixels in one digital character. After this, number of selected objects in the selected image region of license plate is calculated. If the amount of objects and characters is equal on the license plate, this region is selected as license plate (Fig. 3). In another case next region of the image is considered.

\section{LICENSE PLATE CHARACTERS EXTRACTING}

There are several approaches for finding the character bounds. The simplest approach is to use static bounds, assuming that each character of the license plate is located at the same position. For this goal selected on previous stage region with license plate is divided on the equal grid regions, which can include the character. The advantage of this technique is the simplicity and independency from image quality. The disadvantages of this technique are some background in case of selecting the region and the dividing grid in case of shifting the license plate.
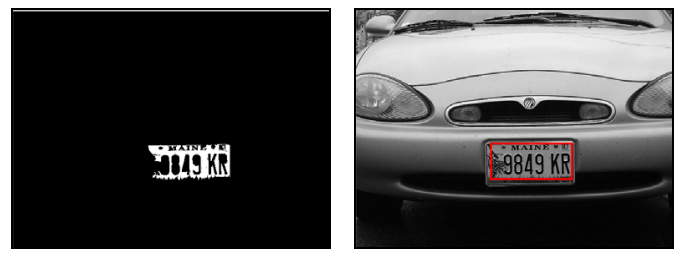

Fig. 3 - Identified region with license plate.

It is possible to use a histogram of summary number of pixels at each column and row of license plate instead static bounds [11]. At this case it is possible to reject some background region from the license plate. Then the peaks and valley indicate on character on license plate. Depending on quality of license plate, this method is very useful since it is independent of character positions. However such technique depends on image quality.

We have proposed the following general algorithm for efficient characters extracting from license plate:

1. to cut square region with license plate received on the previous stage from all source images in order to accelerate execution of proposed algorithm.

2. to threshold cut region using (5) in order to enhance the license plate quality. The value of threshold level is selected taking into account the ration of pixels between background and characters. Such ration is approximately $69 \%$ of background (Fig. 4a).

3. to invert the license plate received on the previous stage (Fig. 4b).

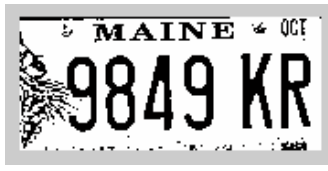

a)

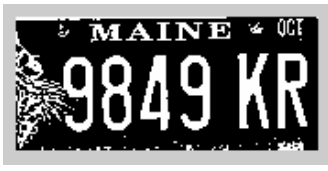

b)
Fig. 4 - Selecting of license plate.

4. to apply the median filter to inverted license plate in order to reject a noise from the template.

5. to renumber each selected region due to the connectivity reason taking into account the following necessary parameters: (i) width of the selected object which is renumbered, (ii) high of the selected object which is renumbered and (iii) total sum of the white pixels in the selected object (pixels selected as 1). Such parameters have to be in proportion with the width, high and 
sum of character of license plate. If the selected object doesn't satisfy the parameters above in boundary of some threshold then the next object is rejected and renumbered (Fig. 5a).

6. to cut the character region with maximum and minimum parameters of high and width for each selected region (Fig. 5b).

7. to resize character region from previous step to the standard parameters of width and high using bicubic interpolation technique in order to use such image in following recognition procedure (Fig. 5c).

\section{RECOGNIZING OF CHARACTERS USING NEURAL NETWORKS}

The character sequence of license plate uniquely identifies the vehicle. It is proposed to use artificial neural

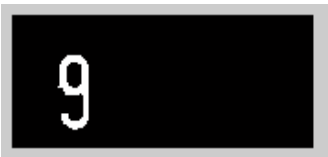

a)

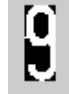

b)

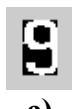

c)
Fig. 5 - Extracting the character from license plate.

networks for recognizing of license plate characters, taking into account their properties to be as an associative memory. Using neural network has advantage from existing correlation and statistics template techniques [12] that allow being stable to noises and some position modifications of characters on license plate.

The images of characters (30x19 pixels) form the training set of neural network. The multilayer neural network is used for character recognition [13]. The training vector of neural network consists of 663 elements (also this is a number of input layer neurons) and it is created by vectors of image rows of the license plate characters in binary format. A supervisor sets the number of output neurons of neural network on the training stage. Each layer of neural network contains the matrix of weight coefficients $W$, vector of thresholds $b$ and vector of output values $a$ (Fig. 6). The output of each layer of neural network is presented by an overhead index and is calculated as following:

$$
\begin{gathered}
a^{1}=f^{1}\left(I W^{1,1} p+b^{1}\right) \quad a^{2}=f^{2}\left(L W^{2,1} a^{1}+b^{2}\right) ; \\
a^{2}=f^{2}\left(L W^{2,1} f^{1}\left(I W^{1,1} p+b^{1}\right)+b^{2}\right) .
\end{gathered}
$$

The neural network showed on Fig. 6 contains $\mathrm{R}^{1}$ inputs, $S^{1}$ neurons of the hidden layer and $S^{2}$ neurons of output layer. The outputs of every intermediate layer are the inputs of the next layer. Therefore, output layer 2 can also be considered as one layer neural network with the $S^{1}$ inputs, $S^{2}$ neurons, and weighted matrix $\mathrm{W}^{2}=\mathrm{S}^{2} \mathrm{xS}^{1}$. The $\mathrm{a}^{1}$ vector is the input to layer 2 with $\mathrm{a}^{2}$ outputs. The same approach is taken for any layer of neural network.

Gradient descent backpropagation method with momentum and adaptive learning rate is used for neural network training [14]. Back propagation is used to calculate derivatives of performance with respect to the weight and bias variables $\mathrm{X}$. Each variable is adjusted according to gradient descent with momentum:

$$
d X=m c * d X p r e v+l r * m c * d p e r f / d X,
$$

where $d X p r e v$ is the previous change of weight or bias, $l r$ is learning rate, $m c$ is momentum constant.

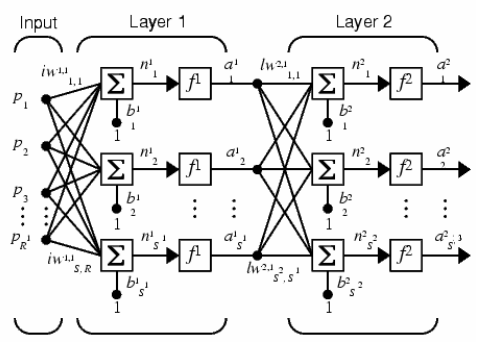

Fig. 6 - Structure of neural network.

For each epoch, if performance decreases toward the goal, then the learning rate is increased by the factor $l r$ inc. If performance increases by more than the factor max_perf_inc, the learning rate is adjusted by the factor $l r \_d e c$ and the change, which increased the performance, is not made. Training process stops when any of these conditions occur: (i) the maximum number of epochs is reached, (ii) the maximum amount of time has been exceeded, (iii) performance has been minimized to the goal, (iv) the performance gradient falls below min gradient, (v) validation performance has increased more than max_fail times since the last time it decreased.

\section{EXPERIMENTAL RESULTS}

The LPR system was researched using software routines designed in Matlab 6.5 [13]. During the researches Fuji MX - 1700 digital camera with resolution of $640 \times 480$ grayscale pixels connected to the computer through USB serial port is used. Such camera allows transferring 25 frames per second.

Feed forward neural network for LPR system is used. The structure of neural network includes input layer with 366 inputs, one hidden layer with 50 neurons and output layer with 46 neurons. Each layer contains log-sigmoid transfer functions. Described above gradient descent backpropagation method with momentum and adaptive learning rate is used for neural network training. Neural network is trained on the quality images showed by 
alphabetic characters with supervisor outputs. Each neural network output shows one possible character, which has to be classified. Neural network is trained for 0.00001 mean square error (Fig. 7).

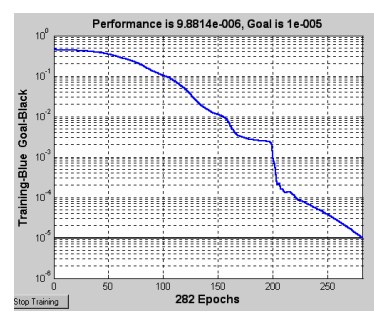

Fig. 7 - Training progress of neural network (left).

In order to interpret the noise and shifting influence on character 'salt \& pepper' noise to the image with uniformly distributed law and different density is added [15]. Noised characters are entered to neural network and classified. The classification results of license plate character recognition are shown on Fig. 8. First column on Fig. 8 shows the ideal images of license plate characters for neural network training. The second column of Fig. 8 shows images noised by 'salt \& pepper' technique, which was entered to neural network in order to be classified. The third column of Fig. 8 shows the result of true or false classification.

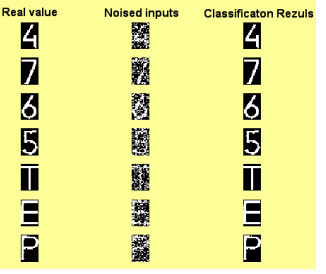

Fig. 8 - Character classification by neural network.

In order to research dependences between noise intensity and possibilities of neural network to classify correctly the license plate characters each character image is noised 50 times with the same noise density and detected the percent of correct recognition (Fig. 9).

As it is seen from Fig. 9 neural network correctly classifies license plate characters with probability $95 \%$ in the presence of $50 \%$ noise density.

\section{CONCLUSIONS}

The purpose of this paper is to investigate the possibility of automatic recognition of vehicle license plate. We can improve quality of the vehicle image using fusion technique, then extract the license plate and isolate characters contained on the plate, and finally identify the characters on the license plate using artificial neural network. The experimental results have shown the ability of neural network to recognize correctly

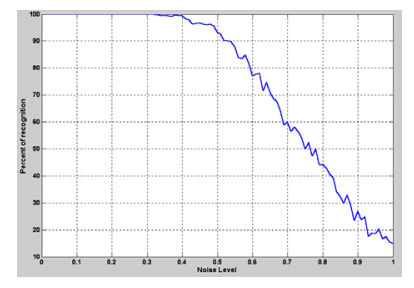

Fig. 9 - Dependence between noise level and correct classification of license plate characters.

characters on license plate with probability of $95 \%$ in presence of noise with $50 \%$ density. The proposed approach of license plate recognition can be implemented by police to detect speed violators, parking areas, highways, bridges or tunnels. Also the prototype of the system is going to be integrated and tested as part of the sensor network being developed by Ayers Island, LLC as part of their Intelligent Island $^{\mathrm{TM}}$ system.

\section{ACKNOWLEDGMENTS}

The authors are grateful for the support of the U.S. Civilian Research and Development Foundation, CRDF Awards TGP-936 and TGP-937, NATO Scientific Affairs Division within the International Project PST.CLG.978744 "Using Sensor Fusion and Neural Network Techniques for Robot Control" and Ministry of Education and Science of Ukraine.

\section{REFERENCES}

[1]V. Kamat, S. Ganesan. An Efficient Implementation of the Hough Transform for Detecting Vehicle License Plates Using DSP'S, Cellular Neural Networks and Their Applications. Proceedings The IEEE 31st Annual 1997 International Carnahan Conference. 1997. pp 209-218.

[2]J.A.G. Nijhuis, M.H. Brugge, K.A. Helmholt. License Plate Recognition Using DTCNNs, Security Technology. 1997. Proceedings 1998 Fifth IEEE International Workshop on Publish. 1998, pp 212-217.

[3]Y. Cui, Q. Huang. Automatic license extraction from moving vehicles. In The Int. Conf. On Image Processing, 3-volume set, 1997.

[4]H. Li, B. Manjunath, S. Mitra. Multisensor image fusion using the wavelet transform, Graphical Models Image Process, 57 (3) (1995), pp 235245.

[5]P. T. Burt, E. H. Andelson. The Laplacian pyramid as a compact image code, IEEE Trans. Commun. 31 (4) (1983), pp 532-540.

[6] P.T. Burt, R.J. Kolczynski. Enhanced image capture through fusion, in: Proceedings of the 4th International Conference on Computer Vision, Berlin, Germany, May 1993, pp. 173-182. 
[7]A. Toet, L. J. van Ruyven, J. M. Valeton. Merging thermal and visual images by a contrast pyramid, Opt. Eng. 28 (7) (1989), pp 789-792.

[8]G. K. Matsopoulos, S. Marshall, J.N.H. Brunt. Multiresolution morphological fusion of $M R$ and $C T$ images of the human brain, Proc. IEE: Vision, Image Signal Process. 141 (3) (1994), pp 137-142.

[9]D. A. Yocky. Image merging and data fusion by means of the discrete two-dimensional wavelet transform, J. Opt. Soc. Am. A: Opt., Image Sci. Vision 12 (9) (1995), pp 1834-1841.

[10] Z. Zhang, R. S. Blum. A categorization of multiscale-decomposition-based image fusion schemes with a performance study for adigital camera application, Proc. IEEE 87 (8) (1999) 1315-1326.

[11] C. Rafael Gonzalez, E. Richard Woods. Digital Image Processing1993, Addison-Wesley, ISBN 0201-60078-1.

[12] B. Kroese. An Introduction to Neural Networks, Amsterdam, University of Amsterdam, 1996, 120 $p$.

[13] J. Stephen Chapman. MATLAB Programming for Engineers, 2nd Edition, Brooks/Cole Publishing Company, 2002.

[14] M. T. Hagan, H. B. Demuth, M. H. Beale. Neural Net work Design, Boston, MA: PWS Publishing, 1996.

[15] S. Jae Lim. Two-Dimensional Signal and Image Processing. Englewood Cliffs, NJ: Prentice Hall, 1990. pp. 469-476.

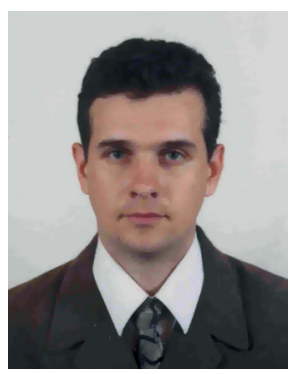

Vasyl Koval was born in Ternopil in 1975. In 1998 he received B.Sc. in "Management Information Systems" at Ternopil Academy of National Economy, Institute of Computer Information Technologies. In 1999 he obtained M.Sc. in "Economic Cybernetics" at Ternopil Academy of National Economy. Since 1999 he is Ph. D. Student in "Computing Machines, Systems and Networks" at Ternopil Academy of National Economy.

Now he works as lecturer of Department of Information Computing Systems and Control of the Institute of Computer Information Technologies of Ternopil Academy of National Economy, Ukraine; scientist of Research Lab. of Automation Systems and Network of Ternopil Academy of National Economy.

His research area includes: Robotic Systems; Distributed Systems; Sensor Fusion Techniques; Sensor Fusion Algorithms; Data Acquisition Systems; Stereo Vision; Stereo Matching; Image
Processing; Artificial Intelligence; Neural Networks; Robot Navigation Systems; Sensor Systems, etc.

\section{Dr. Volodymyr}

0.

Turchenko received his M.S. degree from Brest Polytechnic Institute, Belarus in 1995 and Ph.D. degree from Lviv National Technical University, Ukraine in 2001 both in computer engineering.

Now he is an Assistant Professor of the Information Computing System and Control Department of the Institute of Computer Information Technologies. He is the leader of Neural Network Research Group. His main research interests are intelligent instrumentation, distributed sensor networks and neural networks.

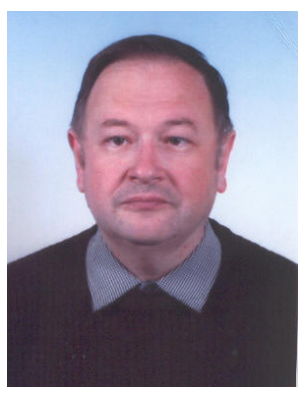

Volodymyr Kochan, Ph.D. of Measurement Engineering, Associate Professor, was born in 1951 in Lviv. In 1973 he received $B$. Eng. Electrical Engineering at Lviv Polytechnic Institute, Ukraine. In 1989 he obtained Ph.D. of Electric and Magnetic Instrumentation at Kiev

Polytechnic Institute, Ukraine.

Now he works as Associate Professor of Department of Specialized Computing Systems and Senior staff scientist of Research Lab. of Automation Systems and Networks of Institute of Computer Information Technologies of Ternopil Academy of National Economy; Department of Computer Science of Ternopil State Technical University; Department of Electrical devices of Technical college of Ternopil State Technical University; Instructor of the practical course on microprocessor application.

His research area includes: Sensor Intelligent System; Distributed Sensor Network; Computer based Intelligent Measurement and Control Systems; Intelligent Controllers for Automated and Robotic Systems in Industry; Sensor Systems Calibration and Verification.

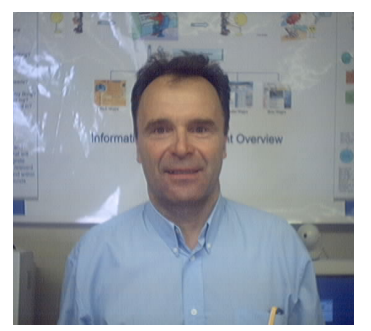

Anatoly Sachenko is Professor and Director of the Institute of Computer Information Technologies, Ternopil Academy of National Economy since 1994. At present he is Visiting Fulbright Professor

at University of Maine, Department of Computer Science for the 2002/03 academic year.

He earned his B.Eng. Degree in Electrical Engineering at L'viv Polytechnic Institute in 1968 and his PhD Degree in Electrical Engineering at L'viv Physics and Mechanics Institute in 1978 and his Doctor of Technical Sciences Degree in Electrical 
and Computer Engineering at Leningrad Electrotechnic Institute in1988. Since 1991 he has been Honored Inventor of Ukraine, since 1993 he has been IEEE Senior Member.

His main Areas of Research Interest are Implementation of Artificial Neural Network, , Distributed System and Network, Parallel Computing, Intelligent Controllers for Automated and Robotics Systems. He has published over 300 papers in areas above.

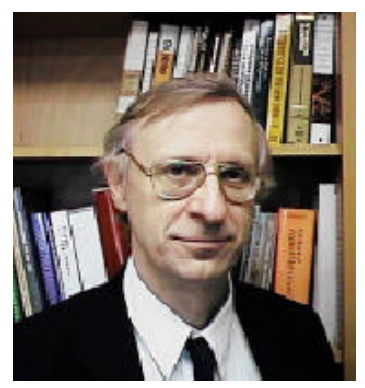

\section{George Markowsky} received his $B A$ in Mathematics from Columbia University and his M.S. and Ph.D in Mathematics from Harvard University. He spent ten years at the IBM Thomas J. Watson Research Center where he served as Research Staff Member, Technical Assistant to the Director of the Computer Science Department, and Manager of Special Projects. He came to the University of Maine as the first Chair of the Computer Science Department. He held that position from December 1983 until August 1989. He served as interim chair for the 1995-96 academic year. He is currently Professor of Computer Science and once again, the Chair of the Computer Science Department.

In August 2001, he became chair of the Mathematics \& Statistics Department in addition to remaining as Chair of the Computer Science Department.

George Markowsky has published 69 papers on various aspects of Computer Science and
Mathematics. He has written an additional 19 technical reports and 6 books on various aspects of computing. He also holds a patent on mechanisms that implement Universal Hashing. His interests range from pure mathematics to the application of mathematics and computer science to biological problems. He has also built voice controlled and enhanced keyboard terminals for use by paralyzed individuals.

George Markowsky served as the President of the Maine Software Developers Association (MeSDA) since its inception in Spring 1993 until May 1998. The Association has grown from 18 members to over 200 and now employs a full-time Executive Director. MeSDA works closely with companies and state agencies to promote the development of the software industry in Maine.

George Markowsky founded a software company, Trefoil Corporation in February 1994. Trefoil Corporation developed the O*NET software for the U. S. Department of Labor that will replace the Dictionary of Occupational Titles. The $O^{*} N E T$ software was released nationally in 1998. In addition, Trefoil has handled tasks ranging from software reengineering and testing, to the marketing of a product called PC-Pedal(tm). Trefoil is currently working on a Phase II SBIR for the National Institutes of Health.

Since that time he has founded several companies, including Ayers Island, LLC, which is developing a research/commercialization complex two miles from the University of Maine on a 63 acre island, and Maine Venture Capital. He is also one of the founders of the Mutli-Sector Crisis Management Consortium. 